Patienten mit progredienter MS helfen

\title{
Intensive Suche nach neuen Wirkstoffen
}

Immunmodulatoren, Vitamine und Statine - Forscher suchen intensiv nach wirksamen Therapien für Patienten mit progredienter MS.

Ein Problem bei progredienter MS sind offenbar neurodegenerative Prozesse, die kaum auf eine klassische Entzündungshemmung ansprechen. So konnten Interferone in Studien mit sekundär progredienter MS (SPMS) zwar überlagerte Schübe verhindern, aber nicht die Krankheitsprogression bremsen.

Nicht besser schneiden bislang auch die neuen MS-Mittel ab. In der Studie INFORMS mit 970 an primär progredienter MS (PPMS) erkrankten Patienten entwickelten die Teilnehmer unter Fingo- limod zwar weniger Schübe und Hirnläsionen als unter Placebo, EDSS-Wert und Hirnvolumen veränderten sich in beiden Gruppen jedoch ähnlich stark, wurde bei der Jahrestagung der American Academy of Neurology in Washington D. C. berichtet.

\section{Kann Laquinimod die \\ Neurodegeneration bremsen?}

Dennoch werden Immunmodulatoren weiter untersucht. Interessant dürften die Ergebnisse der Studie ARPEGGIO mit 375 PPMS-Patienten sein - beim AANKongress als Poster vorgestellt. Darin wird der noch nicht zugelassene Wirkstoff Laquinimod geprüft. Nach ersten Daten bremst die Substanz möglicher- weise unabhängig von der Schubreduktion die Neurodegeneration.

\section{Weitere Ansätze}

Lohnend ist auch der Blick auf ganz andere Ansätze: So wurde auf der US-Tagung eine Studie mit Biotin bei 154 Patienten mit progredienter MS präsentiert. Unter der recht hohen Dosis von $300 \mathrm{mg} / \mathrm{d}$ des wasserlöslichen Vitamins war die Behinderung nach einem Jahr bei jedem achten Patienten deutlich verringert, unter Placebo schaffte dies niemand. Biotin aktiviert die Acetyl-CoA-Carboxylase, ein potenzielles Schlüsselenzym der Myelinsynthese.

Aufhorchen lässt auch die Studie MSSTAT bei 140 SPMS-Patienten: Eine zweijährige, hochdosierte SimvastatinTherapie $(80 \mathrm{mg} / \mathrm{d})$ konnte die Hirnschrumpfung im Vergleich zu Placebo um $43 \%$ reduzieren. Statine dämpfen offenbar entzündungsfördernde Immunreaktionen und behindern die Migration von Leukozyten durch die Blut-HirnSchranke.

(mut)

Lancet 2014; 383: 2213-2221

\section{Paradigmenwechsel in der Therapie}

\section{Aktive MS: Aggressive Therapie rasch erwünscht!}

\begin{abstract}
Patienten mit sehr aktiver MS müssen nun nicht mehr warten, bis die Basistherapeutika versagen, sie können nun auch von Beginn an eine hochwirksame Therapie erhalten.
\end{abstract}

Ausdruck des Paradigmenwechsels in der MS-Therapie in den vergangenen Jahren ist die Zulassung des monoklonalen Antikörpers Alemtuzumab (Lemtrada ${ }^{\circ}$ ) als First-line-Therapie. Voraussetzung für die Therapie ist nur eine klinisch oder per Bildgebung nachgewiesene MS-Aktivität.

\section{Antikörper hallbiert Schubrate}

In den CARE-MS-Zulassungsstudien konnte Alemtuzumab die Schubrate im Vergleich zu Interferon beta-la in etwa halbieren. Und bei 71\% der Patienten hatte sich der EDSS-Wert in Extensionsstu- dien innerhalb von vier Jahren unter der Behandlung mit dem Antikörper entweder verbessert oder stabilisiert.

\section{Risiko-Monitoring ist ein Muss!}

Die meisten Patienten kommen mit zwei Infusionszyklen im Abstand von einem Jahr aus. Allerdings ist ein mehrähriges Risiko-Monitoring nötig, unter anderem auf Schilddrüsenerkrankungen und Nephropathien.

Schon deutlich länger - seit dem Jahr 2006 - wird der Antikörper Natalizumab (Tysabri ${ }^{\circledR}$ ) bei Patienten mit hochaktiver Multipler Sklerose eingesetzt. Eine Infusion mit der Substanz erfolgt alle vier Wochen. In der Studie AFFIRM ließ sich damit die jährliche Schubrate im Vergleich zu Placebo um 68\% reduzieren, und eine anhaltende Behinderungspro- gression trat $42 \%$ seltener auf. Therapielimitierend ist allerdings das Risiko für eine progressive multifokale Leukenzephalopathie (PML). Auch hier ist daher ein enges Monitoring nötig.

Eine Alternative zu den beiden Antikörpern ist Fingolimod (Gilenya $\left.{ }^{\circ}\right)$. Dieser Wirkstoff wird als Tablette einmal täglich eingenommen. In der Studie FREEDOMS konnte Fingolimod die jährliche Schubrate im Vergleich zu Placebo in etwa halbieren.

Und in der Studie TRANSFORMS war die jährliche Schubrate unter Fingolimod um etwa ein Drittel niedriger als mit Interferon beta-1a. Bei Therapiebeginn ist wegen möglicher kardialer Risiken ein EKG-Monitoring nötig.

(mut)

www.aerztezeitung.de 